Roberto Casati

Institut Nicod

CNRS (EHESS-ENS)

\title{
Numerals and word sequences
}

\section{Summary}

According to (Spelke and Tsivkin 2001) numerals are a linguistic and cognitive bridge between two types of "core" knowledge, that is, subitization of small quantities and approximate representation of large quantities. In this paper I go somewhat their way but I also introduce some apriori constraints on what could constitute a bridge. Such constraints are on the 'design' of a numeral system and on its use. The starting point is the consideration that numerals like 'three' (as well as names of days of the week like 'Friday') are non-standard linguistic items. I propose that their peculiarity is primarily neither a syntactic nor a semantic peculiarity. It is instead in their morphology. Mastering numerals and names for days of the week is assigning them a certain non-standard morphology, whereby any numeral is a mandatorily a non-independent part of a longer sequence. It is hypothesized that this nonstandard morphology is associated with a non-standard (at least for language) semantics, i.e. map semantics. In a sense, numerals are an artificial language encroached in natural language. The explanatory advantages of the account are discussed and contrasted with Spelke and Tsivkin 'bridge' account of the role of numerals in cognition.

Numerals. Is there anything special, from the linguistic point of view, about numerals such as 'two' or 'thirty-seven'? On the face of it, they have multifarious, and confusing, syntactic and semantic properties. In 'three is greater than two' numerals appear to function as nouns and to refer to objects. But the objects they purportedly refer to are elusive. ${ }^{1}$ In 'my friends are three', 'I have three friends' or in 'there are three apples on the table' the numeral appears in an adjectival form to express a property of a set ${ }^{2}$ or of a manifold. ${ }^{3}$ However, they are not like standard adjectives (for instance, in many languages in which adjectives can be pluralized, numerals normally aren't). Moreover, the property they purportedly express is mysteriously different from ordinary properties, such as the one expressed in 'my friends are nice'. Finally, sets and manifolds, the purported describees of 'three', are metaphysically very different from each other - here too the semantics of numerals is elusive. ${ }^{4}$

Benacerraf 1965.

Frege 1883

Husserl 1881; Simons 1982.

A difference is assumed here between sets (anstract entities) and manifolds (concrete entities). 
There are specific interesting linguistic properties of numerals. In English there are two main types of numerals: primitive numerals, and compositional numerals. Primitive numerals are those in which no trace of another numeral is to be found. They typically span the initial series of numerals up to the first base ('one' to 'ten'), and then include some other bases ('hundred', 'thousand'). 'Seven' is no more compositional than 'three', but for reasons that will be clear in a while, it is possible to draw a further classification line between small and large primitive numerals. Accordingly, I shall make a threefold distinction here: primitive vs. compositional numerals, and, within the class of primitives, small numerals like 'three' and larger numerals (that is, larger than small ones) like 'seven'. A peculiarity of primitive numerals is that they appear to share properties with a larger class of expressions that includes names of days of the week, of months of the year, of fingers, of letters of the alphabets and of musical notes. ${ }^{6}$ Observe, however, that the analogy only holds for primitive numerals. Names for days of the week, for months, for notes, for letters, are not compositional. You cannot form the expression 'Friday-Tuesday', for instance, to name a day.'

These features of numerals appear to be contingent and idiosyncratic, at least in the sense that they do not flow naturally from intrinsic properties of the numbers (however the latter be construed). There is no metaphysical difference between the number 3 and the number 28 . Surely some explanation is required.

In order to account for these interesting properties of numerals, it has been suggested that attention be paid to their role in number cognition or mathematical cognition at large. The relevant aspects of the research on number cognition are as follows. Two "systems" of "numerosity representation" are generally alleged. The first system represents small numerosities in an exact way. The second system represents large numerosities in an inexact way. The two systems are domain specific: they are triggered by varieties of perceptual inputs that are mutually exclusive. The first system is triggered by collections of up to three-four items; the second system is triggered by larger collections, but not by small collections. The first system delivers a sharp classification of the input: three objects are never categorized as two, two objects never as three. The response of the first system is not only accurate, it is very fast; the system is said to "subitize" cardinality. This means that in order to judge - at the personal level - that there are three objects when we look at the following image: ${ }^{\circ}$ we do not have to count - at the personal level - the items. The second system represents cardinalities in an imprecise way. A collection of 53 items simultaneously presented in vision does not give rise to a sharp classification as is shown by the fact that we are at pain in distinguishing such a collection from a similarly presented collection of 54 items. The two systems do not cooperate or mingle: there is neither an approximate representation of small cardinalities, nor a sharp representation of larger than small cardinalities. Finally, the main purpose of the first system is to deliver absolute representations ('there are three objects here in front of me'); the main purpose of the second systems is to deliver relative representations ('this collection is larger than that').

\footnotetext{
5 Some particular problems posed by 'eleven' and 'twenty' will not be addressed here. Some of the idiosincracies of numeral systems are likely to be independent of the main point of this paper.

$6 \quad$ Bühler 1934 (see Mulligan 1997 :201) was probably the first to remark the analogies between these sequences, which he called 'organizers'. It is interesting to note that names for colors are not on this list, whereas names of musical notes are. It may be that these particular sequences are just an extension of the large class of asymmetric idioms ("black and white", "up and down", "Tom, Dick and Harry").

Compositionality in the field of numerals is not unrestricted, however, as one cannot form expressions such as 'twenty-twenty'. However, look carefully at the various possibilities: 'two-twenty' is acceptable if used to express the hour of the day.
} 
The two modes of operation are documented in adults and, what is more telling, in prelinguistic infants. The latter findings indicate that the systems appear to constitute a form of "initial" or innate knowledge which is, of course, language-independent, as it is manifested before the onset of language.

A methodological point concerning experiments on infants. Experiments on subitizing may elicit hybrid interpretations: part of the interpretation of the results could be based on the experimenter's first-personal assessment of the test display, which may be projected into the infant's capabilities. The fact that the infant reliably distinguishes ${ }^{\circ \circ}$ from ${ }^{\circ \circ}$ in suitably controlled experiments cannot be taken as evidence that the infant distinguishes twoness from threeness without further assumptions about what exactly gets counted or classified (or even perceived). If we fancy to redescribe the input not as a collection of circles but as a collection of couples of possibly non adjacent circles, then the infant is distinguishing threeness from oneness: $\left[\left({ }^{\circ}-^{\circ}\right)\left({ }^{\circ \circ}\right)\left({ }^{\circ}-\right)\right]$ vs. $\left[\left({ }^{\circ}\right)\right] .^{8}$

\section{The role of numerals: a tempting line of thought}

Precision. Imprecise access to cardinality was neglected in traditional discussions of philosophy of mathematics. It is however a key ingredient of numerical cognition. Consider collections whose cardinalities differ by one unit. Subjects are good at determining which is the largest collection between ${ }^{\circ \circ}$ and ${ }^{\circ \circ \circ}$, and are bad at the same performance involving even slightly larger groups of items (say, 35 and 36 items to be perceptually discriminated.) However, if the respective sizes of the large groups are different by a substantial fragment (a Weber fraction, informally: the size of a just noticeable difference is correlated with the magnitude of the stimulus), subjects are again able to make fairly good approximate assessments. ${ }^{9}$ This means that there is a relatively reliable approximate access to numerosity in comparison tasks involving large cardinalities. Observe that it is access to numerosity itself, however approximate, that explains the success in comparison tasks; this is why such access is hypothesized. Access to relative numerosity, whatever that could be, would not constitute an explanation, but a redescription of the performance.

Why all this matters for numerals. In order to understand numerals, we could ask where they enter stage in the development of cognition; what role they could fulfill in view of the data. A tempting line of reasoning is as follows. Consider again the imprecise system, working for large cardinalities. Faced with a collection of 53 items, we would not represent the items as being 53, but as being some indeterminate rather large amount. Our best type of performance would make us detect a difference between that collection and a collection of, say, 43 items. But the comparison would work without us having any sharp idea of the actual cardinality of the terms; provided the difference between the collections is somewhere in the area of the Weber fraction. The tempting line of thought is now this: If we want a sharper, and possibly an absolutely sharp idea of the numerosity of the large collection(s) in front of us all we need is to count the items; now counting amounts to mastering a certain symbolic system. Hence mastering of a certain symbolic system can sharpen our representations, and (possibly) can create sharp representations. The symbol system in question is, of course, the fragment of natural language constituted by numerals.

\footnotetext{
$8 \quad$ Interference between figural properties and subitization has been documented too (Trick and Pylyshyn 1993).

Deahene (1997) for a review of the literature.
} 
Spelke and Tsivkin (2001) suggest that empirical evidence supports in part this line of thought. They take the two number systems as part of our initial endowment of knowledge, and they hypothesize that conceptual development occurs when a cognitive bridge ${ }^{10}$ can be thrown between the world of small cardinalities (the domain of the first system) and the world of larger cardinalities (the domain of the second system), in such a way that precision (a definitory feature of the first system) can reach into the preserves of the second system. Natural language is for Spelke and Tsivkin the bridging factor. Although the details of the bridging are admittedly "obscure"11, it is to be expected that the relevant component of language is the fragment of numerals, and that the relevant features of language are (i) the fact that the language is not domain specific and (ii) that fact that language is a powerful combinatorial system. Being domain a-specific, language can generate representations that apply to entities in both the domain of small collections and the domain of large collections. And the combinatorial aspect of language is the actual engine behind the possibility of linking the two domains. Now, surely the fragment of numerals exhibits the required features (it is domain a-specific and is compositional.) So there is reason to think that it constitutes the required bridge.

The problem with 'seven'. Although there is a theoretical (as well as an intuitive) appeal to the hypothesis that the fragment of numerals is the bridge between two types of cardinality cognition, the hypothesis can be challenged. First, the explanation that mentions the combinatorial system is incomplete. 'Seven' is a primitive numeral - it is not the result of any combination. If we had positional numerals in base three, so that our 'four' were pronounced 'one-one', and seven 'two-one', then it would be immediately possible to think of the combinatorial feature as the relevant link between small cardinalities and not so small cardinalities. But as far as I can ascertain no natural language has a numeral system in base three, and 'seven' is invariably translated into other languages as a primitive numeral. Hence the combinatorial feature of language is not necessary: a primitive numeral such as 'seven' can denote sharply a collection that none of the two non-linguistic systems recognizes with the desired precision.

Put yourself in the shoes of a cognitive designer who is out to find a way to bridge the gap between cognition of small numerosities and cognition of large numerosities. You discover that the resources of the system you are trying to build already include precise knowledge about cardinalities of 1, 2 and 3, in no order. You see that it would be desirable to know with equal precision about larger cardinalities, about which the system has the resources for delivering moderately reliable but imprecise representations. You know that language is flexible, insofar as it is not domain specific and is combinatorial. So you first throw in names such as 'one', 'two', and 'three'. What next? One can see how come you invented 'twentythree'. But how come you invented 'seven'?

Second, and more important, it is disputable that precision is the key factor in bridging the gap between the two systems. Precision in Spelke and Tsivkin's account figures as a normative requirement. As such, it is not required for subitized cardinalities just because these are subitized. And neither is it required for pairwise comparing all types of large cardinalities as some types are comparable just as they are. Precision is a normative requirement only for comparisons of large cardinalities that are very similar to each other. As

\footnotetext{
10 Number cognition is just one example of Spelke and Tsivkin more general hypothesis that natural language bridges different systems of initial knowledge. Another example concerns the integration of space and color representations (see Hermer and Spelke 1996).

$11 \quad$ Spelke and Tsivkin (2001:71)
} 
perceptual cognition does not help in these specific cases, it is just natural that counting comes into play. Hence precision is only required with counting. It is born with counting and does not affect cardinality representations as such. To put the point another way, the precision requirement cannot affect subitized representations, as they already are as precise as it can possibly get. And it does not seem to substantially affect larger cardinality representations, as is proven by the type of persistent mistakes people make in the experiments in which comparison of numerals under time constraints shows effects similar to those of comparison of perceptually presented cardinalities (Dehaene 1997).

\section{The map structure of primitive numeral sequences}

Let us enrich the picture. If learning of small numerals were to be informed by the semantics available to the infant, then it would make no particular sense that small numerals be learned in the sequence 'one-two-three', or in any other sequence, for that matter. As the infant has a representation of threeness available, one could as well teach numerals, that is, introduce them into the language, starting from 'three', and entering 'one' later on. This suggests that reciting the 'one-two-three' rhyme in that particular pattern is an activity that may have little to do with cardinality cognition. However, it seems that the learning sequence is mandatory. No one is taught numerals by being taught countdowns, or any other available order. It follows that learning the sequence of numerals is an activity not only done independently of number cognition; it is also an activity with an independent goal.

More generally, primitive numerals are learned independently of a semantic assignment. You do not even try to introduce 'seven' alone. You spend a lot of time instructing children to learn numerals in the appropriate order. ${ }^{12}$ This effort is comparable to the one of learning a rhyme. But what is this learning effort about? Why invest times and energies in a rhyme?

On top of that, names such as 'one', 'two', 'three', are not introduced independently of the introduction of many other names, such as 'seven' or 'ten'. Why invest time and energy in a rhyme which includes names for numbers the kid has no "initial" access to?

So these are the data: numerals up to 'three' are learnt in a nonrandom way, hence disregarding the fact that their semantics is available to the learner; and they are learnt as ordered parts of a nonrandom sequence of other numerals, disregarding the fact that for these other numerals the learner has no semantics available.

What numerals are, part one: two-dimensional morphology. The proposal I would like to make is that 'three' is not like, say, 'dog' or 'nice', in spite of its occurrence in contexts that are syntactically similar to contexts in which 'dog' or 'nice' may appear (such as 'three is larger than two' or 'I have three friends'), in a new and interesting sense. Where is the difference? The main point is that 'dog' is not part of any other word which is only composed of elements like ' $\mathrm{dog}$ ', ${ }^{\text {, }}$, whereas - this is my contention - 'three' is literally part of 'onetwo-three-four...'. The difference in question is a morphological difference.

One figurative way to describe the difference is to say that locally a sentence like 'I got three apples' has two morphological dimensions. It has the horizontal dimension of spoken

12 Gallistel 1993; Karmiloff-Smith 1992.

13 Hence 'doggy' and 'dooghood' do not qualify as counterexamples. A very strong construal of my claim here is that, actually, 'three' is not a word, and the sequence $\mathrm{S}$ is. 
enunciation, and it also has the unpronounced vertical dimension that inserts 'three' into 'onetwo-three-four...'.

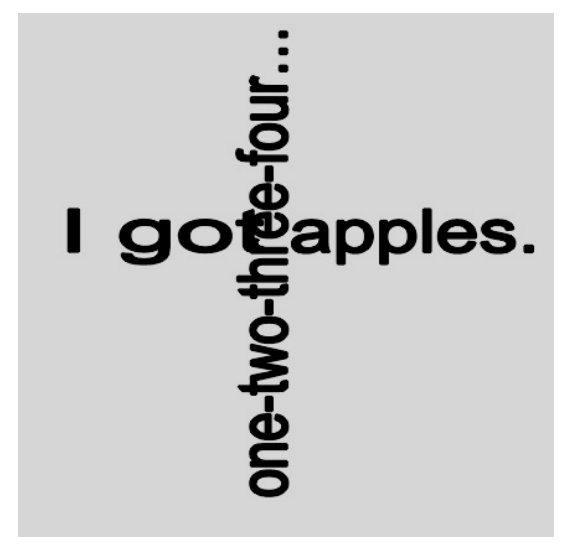

'Three' is then assigned a particular morphology, schematically represented by [PART OF ('three', 'one-two-three-four-five-six-seven-eight-nine-ten')]

I write down the sequence 'three' is part of in full only once; henceforth it will be shortened as ' $\mathrm{S}$ '. ${ }^{14}$ There are two restrictions on what counts as a part of S. First, S is quantized: 'woth', which is a part of 'two-three', is not a part of S in this sense - it is not morphologically visible. Second, only atomic parts are used: 'two-three' is not a part in the intended sense.

What numerals are, part two: Primitive numerals are one map. The second aspect of the thesis I am putting forward is that the phonetic and quantized sequence $\mathrm{S}$ is a map (not in the mathematical and trivial sense of a mapping, but in the more mundane and interesting sense of a road map). S works like a map, has map properties, and being quantized, it works like a map whose spatial resolution is given by the size of the pixel. $\mathrm{S}$ is the vehicle, it is what does the representing. An immediate consequence of the thesis is that the learning effort for numerals is explained in a simple way: it takes time and effort to construct the map, that is, to construct the vehicle for the representation. This is so because the vehicle is one long phonetic sequence, possibly the longest single phonetic sequence the child must learn at this stage.

Maps semantics and map cognition. Assume you already have a map at your disposal; a mundane map, of the type encountered in everyday life. Learning to use a map consists basically of learning to find one's way in space by orienting the map. This capability decomposes into two independent, but interrelated capabilities. It must be understood that the representation vehicle works as a set of designators for locations, where the designation is structured in a specific way; and it must be learnt how to situate the map. Situating the map is a matter of establishing that a point or a region in the space the map represents coincides with (that is, is literally identical to) a point or a region on the map. This is the function typically performed by 'you are here' pointers, together with the non-representational fact that the map is physically nailed to the location that does the situating. Understanding the designation structure (Casati and Varzi 1999; Casati 2001) requires to consider each region of the map as

14 One may use a symbol such as ' $\sim$...' to indicate that there is a word the word replaced for the three dots is a proper part of. 'Three' would then be represented morphologically as ' $\sim$ Three'. A way to express this colloquially is to say that 'three' is syn-morphological. 
a designator for a region in the world, and to see that such designators are structured. In particular, and typically, they obey some part-whole and topological strictures: for instance, a map region $r$ is part of a map region $r$ ' just in case the referent of $r$ is part of the referent of $r^{\prime 15}$, and whenever a map region $r$ is between map regions $r$ ' and $r$ ', the referent of $r$ is between the referents of r' and r',

Back to numerals, once sequence $\mathrm{S}$ has been learnt (that is, it has been stabilized in long-term memory), its properties are so similar to those of a map - albeit of a very simplified, unidimensional map - that the use of S can be taught by appealing to resources from map cognition. These resources include the understanding (i) that in order to go from the referent of a certain map region to the referent of a distinct map region one may have to pass through the referent of a third, further different map region (say, one cannot jump from the referent of 'five' to the referent of 'seven'; one has to go through the referent of 'six'). The resources also include understanding (ii) that the portion of quantized S that includes 'one-two-threefour-five' is on the face of it larger than the portion of S that includes 'one-two'.

These two features of understanding make map-oriented learning of S support an ordinal and a cardinal construal of numerals respectively. This fact may constitute an advantage over a more restrictive hypotheses, according to which numerals are born ordinals or cardinals.

'Friday'. Notice a parallel with names for the days. They are learned as rhymes, are introduced in an ordered way and wholesale, are non-compositional, and once learned, their sequence (call it W, short for 'Monday-Tuesday...') can be literally seen (or figuratively, as one should better say 'heard') to have map properties: they can be situated, one cannot jump from the referent of 'Thursday' to the referent of 'Saturday' without passing through the referent of 'Friday', and finally the span 'Monday-Tuesday-Wednesday' is seen (heard) to be larger than the span 'Monday-Tuesday'. Another parallel holds for names of the letter of the alphabet.

Entering available representations. By drawing a comparison to maps, so far only the semantic structural properties of numerals are assumed to be understood; nothing has been said about the intended semantics. But of course, once the map features of S are understood, the available, automatic semantics for small numerals can be entered. However, the learner need not assign any particular representation to numerals larger than the small numerals. It is enough that the learner be able to use the map, in the sense of being able to operate on it.

What the thesis is not; and some consequences of the thesis. At this point is important to prevent some possible mis-interpretation of the main hypothesis, and to indicate some consequences and predictions.

(i) The thesis is not that all elements of map use and cognition are retrieved or activated in full, but only that some, relevant aspects are taken into account. In fact, there are important disanalogies that block a full assimilation with maps, such as the quantized aspect of numerals and names for days. A closer analogy would be with a pixelized map.

(ii) There is a certain holistic flavor to the idea that numerals are somewhat represented wholesale. However, the thesis is different from the thesis that in order to master the concept of three one must have mastered the concepts of one, of two, or even of seven (in the sense in which it is said that in order to master the concept of a teacher one must muster the concept of

15 Some singular terms in natural language are referentially structured in a part-whole fashion that matches the morphological composition: The referent of 'The county of Exeter' includes the referent of 'Exeter'; however, this is not the general case, as the referent of 'The capital of Italy' does not include, and is rather included in, the referent of 'Italy') 
a pupil). The latter is a semantic thesis I am not endorsing or even suggesting. The idea is instead that we have a tacit morphological representation (the sequence $\mathrm{S}$ ), not a tacit semantic representation. (Constraints on the "processability" of S may be not relevant: it does take time and effort to learn numerals.)

What are the consequences? According to the thesis, numerals and names for the days of the week are small "artificial languages" encroached into natural language. The thesis is compatible with the claim that written numerals may have predated spoken numerals. ${ }^{16}$ For if numerals are not part of language, and are incorporated elements, where do they come from in the first place? The answer may be that they got incorporated into spoken language from written language or from iconic representations. If not written language, it could just have been gestures. In either case, we learned to pronounce a visual image. Primitive numerals are a map that you can pronounce. This would be an interesting case in which spoken language is parasitic on something else - upon non-spoken language or upon other public representational instruments. $^{17}$

The account is also compatible with the fact that some cultures use names of body parts as numerals. In point of fact, body parts are not assigned randomly to numbers. Hence the sum of all body parts used as numerals can well be used as a map in the proposed sense.

On compositional numerals and their representations. According to Spelke, people form an analog cardinality representation (not necessarily consciously accessed) when they listen to words like 'fifty-six'. The representation in question is approximate, which explains why people are faster and more accurate in processing 'is fifty-six larger than forty-three?' than they are in processing 'is fifty-six larger than fifty-eight?' (Dehaene et al. 1999). However, for the actual use of 'fifty-six' (not in an experimental setting, not under fast response constraints) the induced analog representation is totally irrelevant. ${ }^{18}$ It must be irrelevant, because it is imprecise. The mental proxy of the verbal (pronounceable) 'fifty-six' is not oneto-one correlated with the verbal 'fifty-six', as it could fit the verbal 'fifty-seven'. Moreover, the verbal 'fifty-six', when it is used, does not make "numerosity cognition" more precise, as it cannot: numerosity cognition in non-very-small numbers is imprecise.

Compare, for example, how calculations about time spans based on days of the week are relatively clumsy. Our "intuitions" about the number of days between today Tuesday and Friday in two different weeks are confused ${ }^{19}$ and are not improved by our acquaintance with subtracting or adding by using numerals. Acquaintance with numerals simply did not improve our numeric cognition. We have instead to assign dates to the days, and then subtract and possibly add by explicitly using the public numerals. Possibly some training could modify this state of affairs; but this would not amount to creating new, stable "representations".

'Seven' as a by-product of constraints on the learnability of maps: a speculation. Primitive but large numerals like 'seven' extend the knowledge of small numerals into the next immediately accessible field. It is precisely because 'seven' is close enough to 'three' that it

\footnotetext{
16 Boyer, 1991, although this is only a speculation so far as I can ascertain. Another problem concerns the use of numerals in illiterate cultures; one should check where they got their numerals from, for instance from a nearby culture that it turn incorporated it from written language.

17 It is possible that spoken language incorporates many other non-linguistic elements, pieces of the external world that we learned to pronounce. Possibly demonstratives are such an incorporation - pronounceable gestures.

19 An old Wittgensteinian lesson.

This is anecdotal evidence in need of empirical confirmation.
} 
could be grasped easily. At the same time, why invent 'seven' in the first place? Why aren't numerals immediately compositional after 'three', which would be the perfect solution to the design problem evoked by Spelke and Tsivkin, of bridging the gap between two initially available systems of numerosity cognition? The solution may come from the map nature of numerals. A narrow, "contingent", "historical" account would have it that as primitive numerals typically span from 'one' to 'ten', possibly to some translations of 'twenty' in other languages, they are likely to be born with the use of fingers, possibly both fingers and toes, for counting. Hence names were needed for the whole set of fingers (or were possibly derived from antecedent names for fingers. ${ }^{20}$ ) However, 'seven' could still have been replaced by a compositional numeral created out of the small primitive numerals. A more "structural" account may address this problem. Maps properties are readable only off items that are sufficiently rich. Although the part of S including only 'one-two-three' does have all the features that can be ascribed to a map (part-whole structure, and mandatory passage through the intermediate regions), still these may be much too poorly exhibited in the map to elicit learning of them. The 'one-two-three' sequence would be too small. A larger sequence would support the generalizations that are necessary to understand the map structure of the sequence. The invention of 'seven' is vindicated.

\section{Conclusions}

The account proposed here is that the sequence of primitive numerals is represented morphologically as a single unit ${ }^{21}$ and that this large unit is partly interpreted the way a map would be.

Some consequences of the thesis have been hinted at: the learning effort for numerals is explained in a simple way, as it takes time to memorize the long sequence; the account is compatible with known aspects of map cognition; it is compatible with the possibility that written numerals have been introduced before spoken numerals; and with the fact that in some cultures numerals are represented by body parts. Furthermore, the map interpretation supports both ordinal and cardinal construals of numerals, and makes a plausible place to the invention of 'seven'. Finally, the account is general insofar as it also covers other non "numeric" examples, such as names of days of the week, of months, of fingers, of letters of the alphabet, of musical notes. Numerals are in no way special.

Against Spelke and Tsivkin, numerals are not what makes it possible to improve number cognition. They are all there is as the next step in number cognition. Put otherwise, there is no number cognition on top of the first, subitizing step, and there is no number cognition for large numbers in the sense that the approximate system cannot be "made" precise by the use of numerals. 57 is invisible to cognition. In this deflationary sense the present account resonates with Wittgensteinian views: "Our language can be seen as an ancient city: a maze of little streets and squares, of old and new houses, and of houses with additions from various periods; and this surrounded by a multitude of new boroughs with straight regular streets and uniform houses" (Philosophical investigations, 18) However, the sequence of primitive numerals does not fall in either category. It is neither a clumsy part of the old city, nor a neat part of the suburbs. It is like a fallen deity, a beautiful crystal placed in the very center of our hamlet.

20 Names of fingers and toes are interesting in themselves as they constitute sequences. Note that pianists have to relearn names for fingers as numerals.

$21 \quad$ Classifying $\mathrm{S}$ as a unit is, of course, relatively loose talk. It is not clear that $\mathrm{S}$ is a word. 


\section{References}

Benacerraf, P., 1965, "What numbers could not be", Philosophical Review, 74:47-73, 1965.

Boyer, C.B., 1991, A History of Mathematics, 2nd Ed., Revised by Uta C. Merzbach. NY:

Wiley.

Bühler, K., 1934, Sprachtheorie. Jena: Fischer.

Casati, R., 2001, "Formal Maps" (MS).

Casati, R., Varzi, A.C., Parts And Places. Cambridge, Mass.: MIT Press, 1999.

Dehaene, S., 1997, The number sense. Oxford: Oxford University Press.

Dehaene, S., Spelke, L., Pinel, P., Stansecu, R., Tsivkin, R., 1999, "Sources of mathematical thinking : behavioral and brain-imaging evidence". Science, 284, 970-974.

Frege, G., 1883, Grundlagen der Arithmetik, eine logisch-mathematische Untersuchung über den Begriff der Zahl. Breslau

Gallistel, C.R., 1993, The organization of learning. Cambirdge, Mass.: MIT Press.

Hermer, L., Spelke, E. S., 1996, Modularity and development: the case of spatial

reorientation. Cognition, 61, 195-232.

Husserl, E., 1891, Philosophie der Arithmetik. Halle: Pfeffer.

Hurford, James R., 2001, "Languages Treat 1-4 Specially", Mind and Language, 16, 69-75.

Karmiloff-Smith, A., 1992, Beyond Modularity. Cambridge, Mass.: MIT Press.

Mulligan, K., 1997, “The Essence of Language: Wittgenstein's Builders and Buhler's

Bricks", Revue de Métaphysique et de Morale, 2, 193-215.

Simons, P., 1982, "Number and Manifold", in Smith, B. ed., Parts and Moments. Studies in Logic and formal Ontology, München: Philosophia Verlag, 160-199.

Spelke, E. S., Tsivkin, S., 2001, "Initial knowledge and conceptual change: Space and number", in Bowerman, M., Levinson, S., eds., Language acquisition and conceptual development, Cambridge, UK: Cambridge University Press, 70-97.

Trick, L.M., Pylyshyn, Z.W., 1993, What enumeration studies can show us about spatial attention: evidence for limited capacity preattentive processing. J Exp Psychol Hum Percept Perform; 19(2):331-51.

\section{Acknowledgments}

Thanks to Luca Bonatti, Valentina Gliozzi, Pierre Jacob, Nirmalangshu Mukherji, Marco Panza, Achille Varzi, for useful comments on earlier versions of this article. And of course thanks to Kevin Mulligan, who got so many projects on the right track. 